\title{
Deblurring approach for motion camera combining FFT with $\alpha$-confidence goal optimization
}

\author{
Lve HUANG ${ }^{1,2}$, Lushen WU ${ }^{1 *}$, WenYAN XIAO ${ }^{3}$, QINGJin PENG ${ }^{4}$ \\ ${ }^{1}$ School of Mechanical and Electrical Engineering, Nanchang University, \\ Nanchang, 330031, China \\ ${ }^{2}$ College of Applied Science, Jiangxi University of Science and Technology, \\ Ganzhou, 341000, China \\ ${ }^{3}$ Jiangxi University of Science and Technology, \\ Nanchang, 330013, China \\ ${ }^{4}$ Department of Mechanical Engineering, University of Manitoba, \\ R3T2N2, Canada \\ *Corresponding author: 1swu@ncu.edu.cn
}

\begin{abstract}
Sharp images ensure success in the object detection and recognition from state-of-art deep learning methods. When there is a fast relative motion between the camera and the object being imaged during exposure, it will necessarily result in blurred images. To deblur the images acquired under the camera motion for high-quality images, a deblurring approach with relatively simple calculation is proposed. An accurate estimation method of point spread function is firstly developed by performing the Fourier transform twice. Artifacts caused by image direct deconvolution are then reduced by predicting the image boundary region, and the deconvolution model is optimized by an $\alpha$-confidence statistics algorithm based on the greyscale consistency of the image adjacent columns. The proposed deblurring approach is finally carried out on both the synthetic-blurred images and the real-scene images. The experiment results demonstrate that the proposed image deblurring approach outperforms the existing methods for the images that are seriously blurred in direction motion.
\end{abstract}

Keywords: image deblurring, fast motion camera, confidence goal optimization, fast Fourier transform, high-railway defect detection.

\section{Introduction}

Image acquisition is one of the most ubiquitous activities in the information society. As the camera motion during exposure time may degrade the quality of acquired images, image deblurring is an important process in machine vision systems, such as for car-mounted camera, unmanned vehicle, cell phone camera and a variety of industrial inspection cameras [1]. Image deblurring methods differ in their performances, blur 
types, robustness and precision $[\underline{2}, \underline{3}]$. Each method has its own specialty as well as limitation, and there is no solution yet to uniformly address all these issues. Since the serious blur problem is common in the fast motion shooting such as the rail-defect detection system, it is urgent to solve the motion blur for image acquiring system.

When a camera detection system moves along the object at a high speed, the acquiring images would be blurred due to various factors, such as camera motion, defocusing, and atmospheric turbulence under low-lighting conditions. With stable camera system and adequate light source, the exposure in a long time makes the camera sensitive to the motion, which may result in visibly blurred images [4-6]. Therefore, the motion blur is a common incentive to the image degradation in camera motion systems.

Most of the existing methods consider a blurred image model as the convolution of an unknown original image with an unknown point spread function (PSF). Usually, the blind-image deconvolution is conducted by an unknown PSF, which makes the deblurring process too complicate to recover the original image from the only blurred image [7]. A motion deblurring method is proposed with two steps that estimate the PSF and optimize the deconvolution for a sharp image.

To obtain an accurate PSF, the spectral stripe projection and the fast Fourier transform (FFT) are combined with the ability of the spectrum to suppress additive, signal -independent, Gaussian observation noise $[\underline{8}, \underline{9}]$. Then the blurred image is deconvolved by the Richardson-Lucy (RL) method [10] after obtaining the PSF, but the direct deconvolution of the RL will appear in artifacts at the restoration image. Thus, we construct a prediction boundary to promote the convergence of the deconvolution, and an $\alpha$-confidence goal optimized model to reduce the artifacts.

\section{Related research}

It has been proposed to reconstruct sharp images from blurred ones in various existing algorithms of image deblurring. The non-blind image deblurring techniques are such as RL deconvolution [10], Wiener filtering [11], and regularized methods [12]. The RL method restores noise-free or small-noise blurred images based on Bayes' theorem at first. The direct deconvolution of the RL method will appear in artifacts at boundaries or near edges, so various boundary-condition-based deconvolution methods have been proposed to reduce boundary artifacts in the image deconvolution $[13,14]$. The Wiener filter estimates an ideal image with the minimum-mean-square error from a blurred image with the random noise. The regularized methods can minimize the least-squares error between the estimated images and true ones with a certain degree of the image over-smoothness.

Recently, blind-image deblurring has attracted a lot of attentions [2, 15]. For the blind deblurring, both of the PSF and the original image should be estimated from a single blurred image. Existing blind-image deblurring methods can be roughly divided into two categories, variational Bayes (VB) [르, 16] and maximum a posteriori (MAP) [17]].

The VB methods consider all possible solutions instead of the most probabilistic option, thus these methods are time-consuming. The MAP methods seek the most plau- 
sible solution by maximizing the posterior probability using a single global descriptor. The main disadvantage of the MAP is that a blurred image exhibits Gaussian characteristics to a certain extent, which leads to ineffective global minima. Therefore, improved methods are constantly proposed in papers.

For motion image deblurring, LEviN et al. [17] estimated the PSF with a priori knowledge of the natural image gradient obeying the Laplacian distribution to build the MAP framework. KRISHNAN et al. [16] constituted a normalized sparsity measure and then estimated the PSF and the sharp image through the scale-invariant regularization. XU et al. [18] proposed a generalized and mathematically sound $L_{0}$ sparse expression for motion deblurring. Sun et al. [19] analyzed the non-uniform motion blur with a deep learning approach. HAN and KAN [20] calculated the blur kernel by the half -quadratic penalty method and obtained a restoration image by the alternating direction method of multipliers (ADMM).

The above-mentioned deblurring approaches were investigated under different scenarios and assumptions, that is to say, different deblurring models should be constructed according to different blur types.

\section{PSF estimation}

Motion deblurring techniques are oriented toward modeling the degradation image as the convolution of the original image and the PSF, thus applying the inverse process in order to recover the original image. In the blind-image deconvolution, both the PSF and the original images are unknown, and these unknowns must be estimated from a single blurred image. The relation between the blurred images and the original ones can be modeled in two dimensions as follows:

$$
b(x, y)=g(x, y) * h(x, y)+n(x, y)
$$

where $b(x, y), h(x, y)$ and $g(x, y)$ represent the blurred image, the PSF and the original image, respectively. The symbol $*$ is the convolution operator, and $n(x, y)$ is the random noise. The goal of image deblurring is to recover $g(x, y)$ from $b(x, y)$ without the specific knowledge of $h(x, y)$. As the motion blur, $h(x, y)$ can be constituted by blur direction $\theta$ and blur length $L$ as follows $[\underline{6}, \underline{8}, \underline{17}]$ :

$$
h(x, y ; L, \theta)= \begin{cases}\frac{1}{L}, & \sqrt{x^{2}+y^{2}} \leq L \quad \text { and } \quad \frac{x}{y}=-\tan \theta \\ 0, & \text { otherwise }\end{cases}
$$

With Fourier transform, Eq. (1) can be rewritten as

$$
B(u, v)=G(u, v) H(u, v)+N(u, v)
$$

where $B(u, v), G(u, v), H(u, v)$ and $N(u, v)$ represent blurred image, original image, $\mathrm{PSF}$ and random noise, respectively. 


\subsection{Estimation of the motion-blur direction}

The PSF can be estimated by the Hough transform, the steerable filter methods, or the Radon transform $[\underline{8}, \underline{9}, \underline{21}]$. To find the blur direction, there are some methods like Radon transform, Hough transform or any other orientation extraction methods. The Radon transform can accurately predict the motion-blur direction from the experiments, even at long blur lengths. Thus, the Radon transform is chosen for the blur direction detection.

\subsection{Estimation of the motion-blur length}

There are widely used methods $[\underline{21}, \underline{22}]$ to estimate the blur length, such as cepstral, bispectrum and Radon transform. CHANG et al. [9] estimated the blur parameters by inspecting the zero crossings in the "central slice" of the bispectrum, which shows that bispectrum is less variant with noise in comparison to cepstral. WANG et al. [22] calculated the distance between two adjacent dark strips with the bilinear interpolation method for a subpixel-level blur length. The above two methods estimated blur parameters using Fourier transform once, and the estimated precision is not enough. Therefore, to enhance the precision is urgent especially in the serious blur. By performing the FFT twice can obtain the obvious character, thus the improved approach is as follows.

After finding the motion direction $\theta$, the direction can be processed in the horizontal direction by rotating angle $\theta$. Assuming the motion is mostly composed of the translational movement and the distance from the camera to the object is constant, therefore, a forward-direction motion model can be built as follows:

$$
h(x, y)= \begin{cases}\frac{1}{L}, & -L<x<L \quad \text { and } \quad y=0 \\ 0, & \text { otherwise }\end{cases}
$$

When the motion direction is along the $x$ direction (horizontal), the Fourier transform of the PSF is

$$
H(\omega)=\int_{-\infty}^{+\infty} h(x) \exp (-j \omega x) \mathrm{d} x=\frac{2 \sin (\omega L)}{\omega L}
$$

There are obvious multiple peaks after performing the Fourier transform on images, as shown in Fig. 1d but not in Fig. 1b, which depicts the spectrum distribution of the original and the blurred images of Lena, respectively. This character was adopted in the previous methods $[\underline{9}, \underline{22}]$, although it is not very obvious, and the estimation of the blur lengths may have some errors from experiments. Thus, we perform the FFT once more for the precise blur estimation.

Then $H(\omega)$ transforms to $h^{*}(x)$

$$
h^{*}(x)=\ln \left|\frac{2 \sin (x L)}{x L}\right|=\ln (2 \sin |x L|)-\ln |x L|
$$




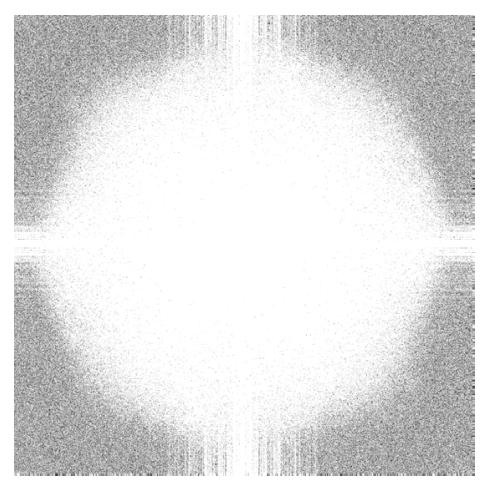

a
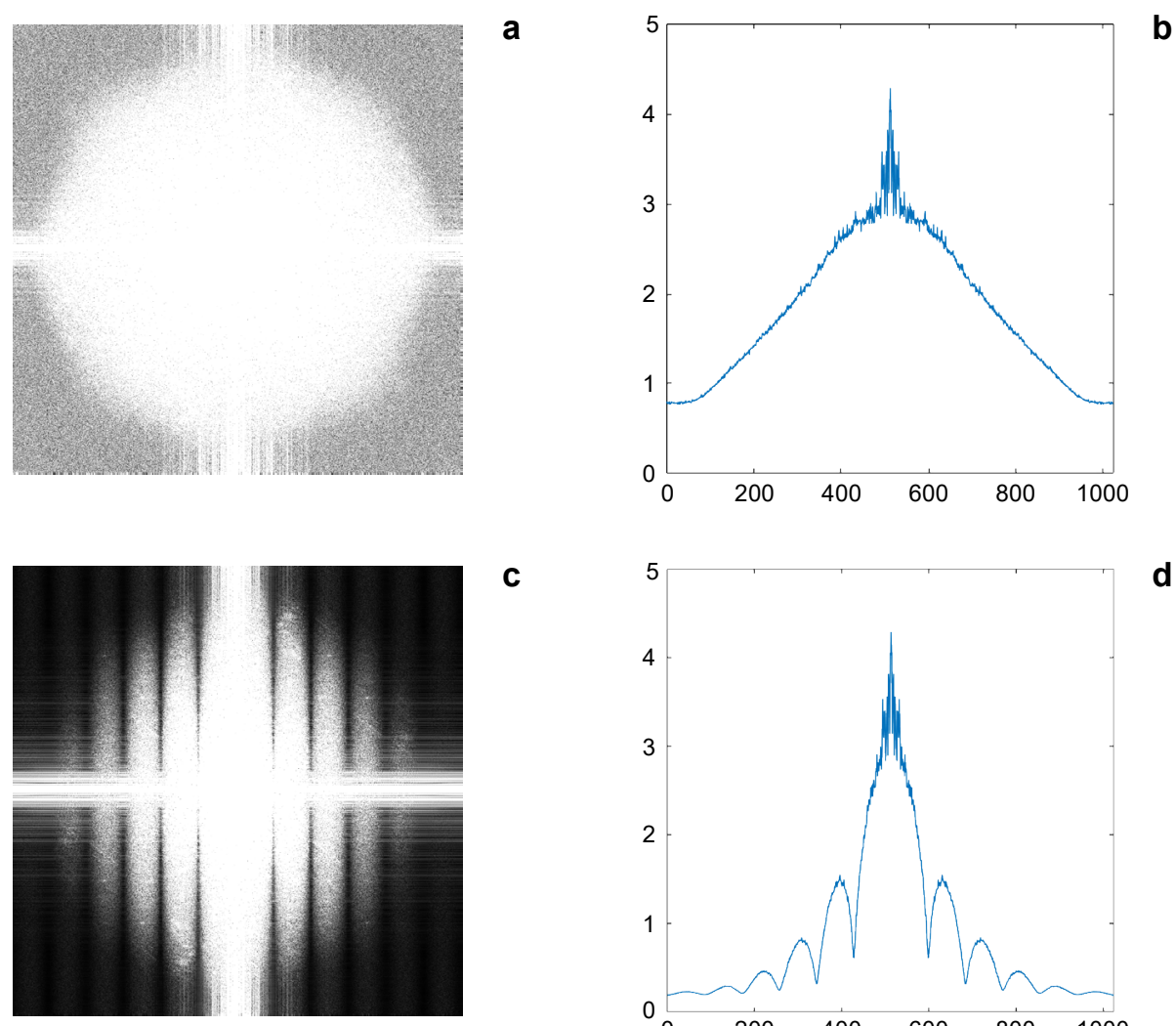

C

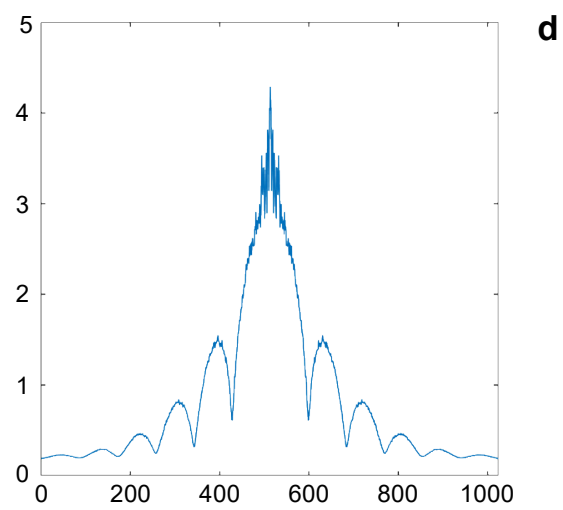

Fig. 1. Fourier frequency spectrum of the original and the blurred images of Lena. 2D FFT of the original image (a), and its column-cumulative of greyscale projection (b). 2D FFT of the blurred image (c), and its column-cumulative of greyscale projection (d).

The fifth order Fourier series expansion of $h^{*}(x)$ in the range $(-\pi / L, \pi / L)$ is expressed as,

$$
h^{*}(x) \approx 1.179+0.1128 \cos (2 L x)+0.0260 \cos (4 L x)+\ldots
$$

For obtaining further obvious character, Eq. (7) is converted into Eq. (8) by performing the Fourier transform again, it becomes

$$
\begin{aligned}
H^{*}(\omega) & =\int_{-\infty}^{+\infty} h^{*}(x) \exp (-j \omega x) \mathrm{d} x \\
& \approx 7.4078 \delta(\omega)+0.3544 \delta(\omega \pm 2 L)+0.0816 \delta(\omega \pm 4 L)
\end{aligned}
$$

$H^{*}(\omega)$ produces values at the integer multiples of $2 L$, and the others are zeros from the $H^{*}(\omega)$ function. Meanwhile, this result is verified by executing the FFT again on Fig. 1d. Figure 2 depicts these outcomes with different blur lengths. The values ob- 

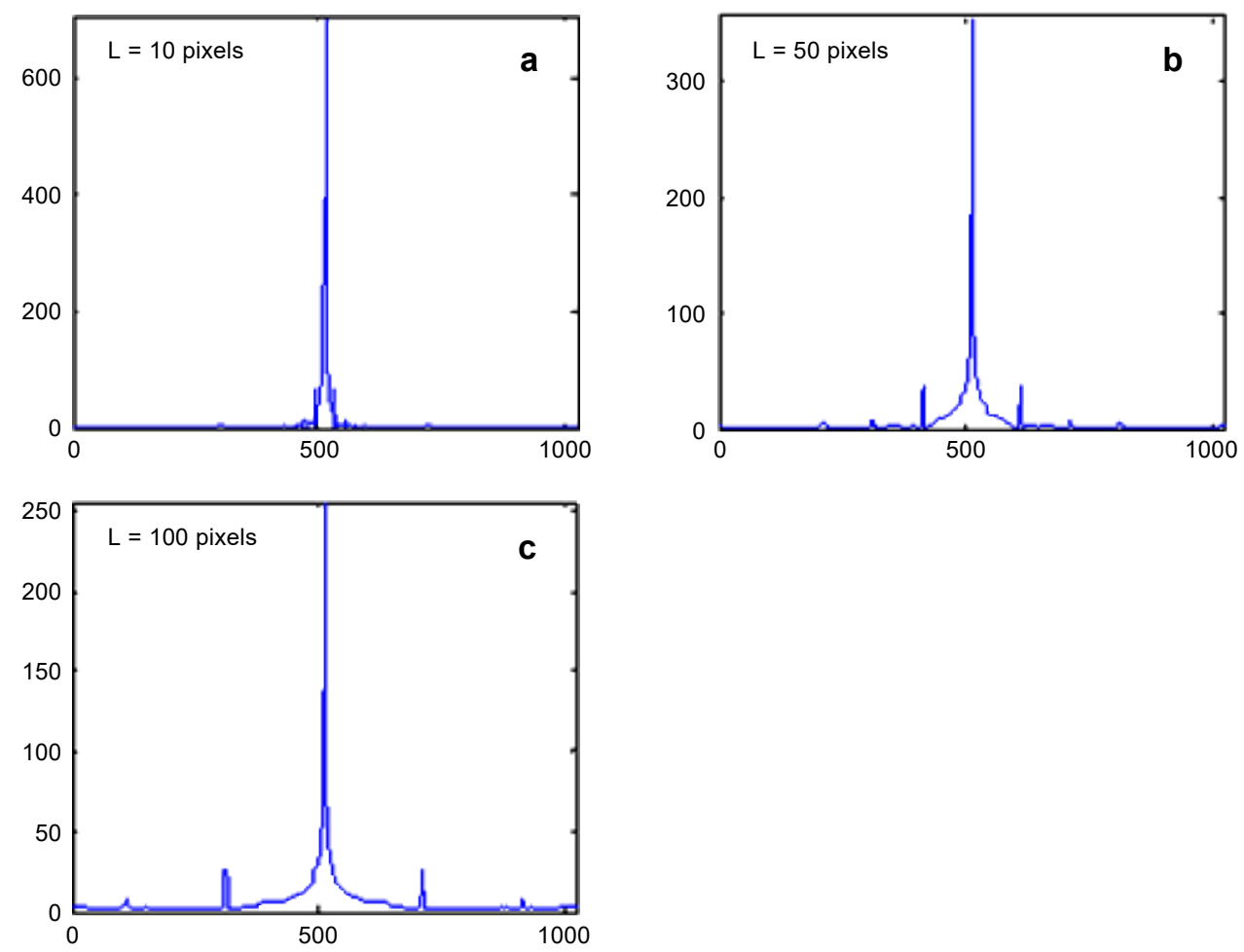

Fig. 2. Regular distributions of the blurred images with different blur lengths: 10 pixels (a), 50 pixels (b), and 100 pixels (c).

tained from the Fourier series expansion of Eq. (8) are consistent with the experimental results obtained from Fig. 2, which proves that the results obtained by the FFT twice are correct and reliable. Comparing with Fig. 1d for the unobvious peaks distribution, Fig. 2 owns the regular pulses distribution especially in the large blur lengths (as shown in Fig. 2c). Then, this obvious pulse character can be easily used to estimate the blur length, especially the large blurs.

Figure 2 shows regular pulse signals, which locate at the positions corresponding to integer multiples of blur lengths. Thus, the distance between the adjacent pulses is a half of the corresponding blur length.

\section{Image deconvolution and $\alpha$-confidence goal optimization}

\subsection{Boundary prediction model}

It is described that the deblurring image can be acquired with the deconvolution if the PSF is known, however the direct deconvolution of real blurred images will result in artifacts, as shown in Fig. 3d, because the deconvolution is an ill-posed problem [1- $\underline{3}]$. Artifacts often appear as wavelike patterns parallel to sharp discontinuities. YUAN et al. [23] 

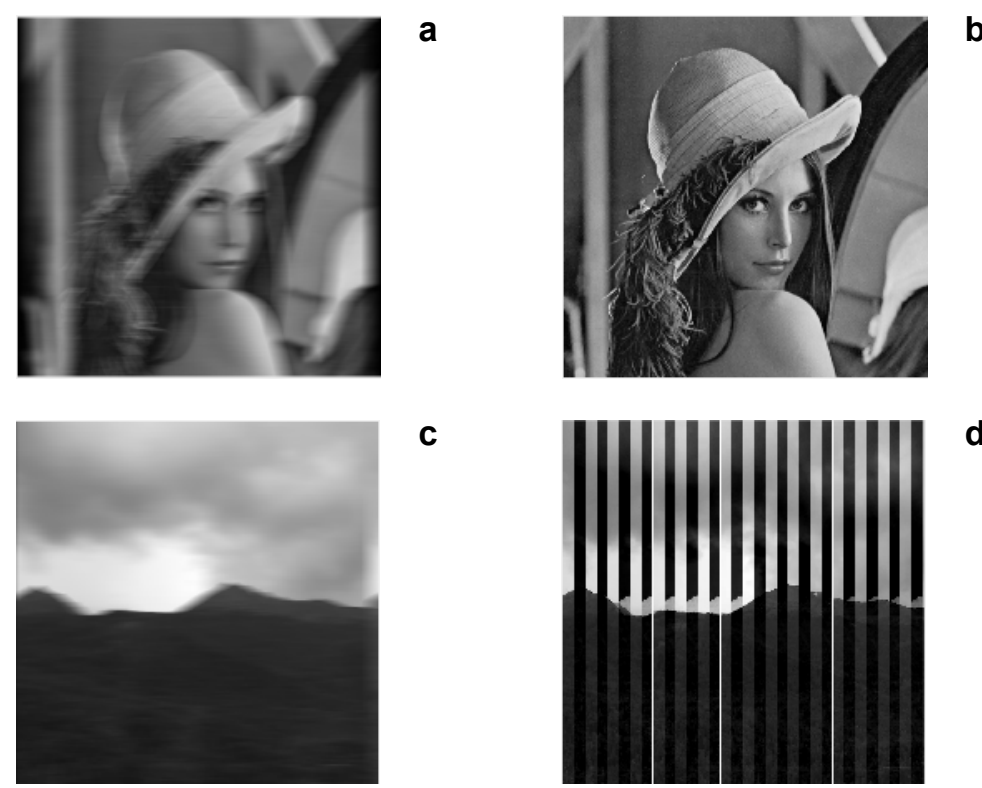

C

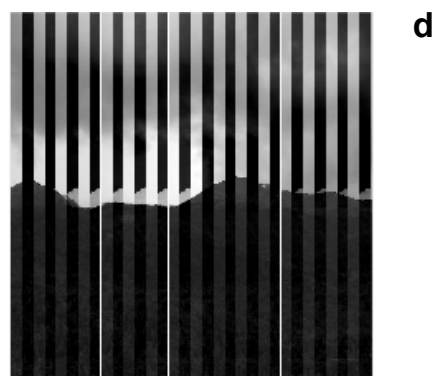

Fig. 3. Artifacts of synthetic and real image deconvolutions. Synthetic blurred image by convolution (a), direct deconvolution image from $\mathbf{a}(\mathbf{b})$, real image $(\mathbf{c})$, and direct deconvolution image from $\mathbf{c}(\mathbf{d})$.

assumed that such light and dark ripples appear mainly due to Gibbs phenomena. SHAN et al. [24] concluded that the artifacts are caused by the observed image noise and the PSF estimate errors, instead of the Gibbs phenomena.

To analyze the cause of the artifacts in Fig. 3d, a lot of comparison experiments have been performed in research. Because a black border appears along sides in the synthetic convolution image such as in Fig. 3a but not in the real image such as in Fig. 3c. The regions outside the boundary in Fig. 3a were assumed to contribute no signals to the synthetic convolution process. In practice, however, there are signals appearing in the real image boundary such as in Fig. 3c. Thus, the deconvolution directly of the real image shows more artifacts than the synthetic image [드-2 28$]$.

Consequently, a suitable image boundary block is predicted before deconvolution at the left and the right image borders, namely $S_{1, M \times L}(x, y)$ and $S_{2, M \times L}(x, y)$, as described in Algorithm 1 (see page 193). Therefore, the calculation dimensions of deconvolution are reduced from $M \times N$ to $2 \times M \times L$, since the blur length $L$ is far less than the image width $N$. Meanwhile the variables $S_{1}$ are reduced, and the convergence of the deconvolution is accelerated.

\subsection{Optimization algorithm}

It is apparent that the greyscale between adjacent columns in nature sharp images show the greater consistency and less difference from the greyscale statistic, because most nature images have spatial correlation with similar greyscale values in most neighboring 
pixels $[\underline{16}, \underline{17}]$. However, this nature statistic feature is different in the blurred image, thus an image restored model based on this statistic feature is proposed as follows.

First, an index of squares sum of the adjacent-column greyscale-difference is described as

$$
R_{i, j+1}=\sum_{x=0}^{M-1}\left[C_{i+1}(x)-C_{i}(x)\right]^{2}
$$

where $C_{i}(x)$ is the greyscale at the $i$-th column and the $x$-th row.

In reality, the consistency and the difference coexist in adjacent pixels of the nature sharp image, but the image with artifacts is different with this feature, therefore this feature is described as the quality assessment index of the deconvolution image by the confidence level. Confidence level quantifies the level of confidence that the parameter lies in the interval $[\underline{29}, \underline{30}]$. In this paper, the $\alpha$-confidence is used as the credibility of adjacent columns in the image greyscale. Then, given the confidence mean $\alpha$ and error permissible value $k, N_{\alpha}$ is a set of the rest pixels that exclude $\alpha \%$ pixels in every column of the whole image,

$$
\mu_{\alpha}(i, i+1)=\sqrt{\sum_{x \in N_{\alpha}}\left[C_{i+1}(x)-C_{i}(x)\right]^{2}}
$$

The adjacent column-consistency measure $(\mathrm{ccm})$ function is

$$
\operatorname{ccm}(i, i+1)= \begin{cases}\mu_{\alpha}(i, i+1)-k, & \mu_{\alpha}(i, i+1)>k \\ 0, & \mu_{\alpha}(i, i+1) \leq k\end{cases}
$$

The column-consistency measure (CCM) index of the whole image is given by

$$
\mathrm{CCM}=\sum_{i=0}^{M-2}[\operatorname{ccm}(i, i+1)]^{2}
$$

Thus, the deconvolution optimization is transformed to a goal optimization with $M \times L$ dimension, and the optimal model is

$$
\min \mathrm{CCM}=\sum_{i=0}^{M-2}[\operatorname{ccm}(i, i+1)]^{2}
$$

Algorithm 1 (see page 193) can optimize the deconvolution image with the $\alpha$-confidence goal optimization, where the inverse process is based on the RL algorithm. In Algorithm 1, the parameter $\beta$ is the synthetic weight of image gradient and linear estimation, its experience value is between 0.2 and 0.8 . According to experiments, we chose the value of $\beta$ as 0.6 .

Experiment results depend on the initial value to some extent, since the optimized model is a high-dimensional and nonlinear matrix. The initial value is selected accord- 
ing to the image convolution principle of blurring process. During the image convolution process, two image boundary blocks with the same length as the blur length are generated on both sides of the original image. Those boundary blocks is the initial value of the model calculation. Therefore, the initial value of variable $S_{1}$ is adjusted to achieve the best restoration automatically by combining the gradient with a linear estimation in Algorithm 1. The initial value of model is the initial value of the image gradient and the linear accumulation. The initial value of the gradient is the pixel difference of adjacent columns, i.e., $b_{i, j+1}-b_{i, j}$, and the initial value of the linear accumulation is $j b_{i, 1} / L$.

Algorithm 1. Image restoration based on the $\alpha$-confidence goal optimization.

Input: blurred image $b$, blur length $L$

$$
\begin{aligned}
& S_{1_{i, j}}=\beta\left(b_{i, j+1}-b_{i, j}\right)+(1-\beta) \frac{j}{L} b_{i, 1}, \quad j<L ; \\
& S_{2_{i, j}}=\beta\left(b_{i, M-L+j}-b_{i, M-L+j-1}\right)+(1-\beta) \frac{L-j}{L} b_{i, M}, j<L ; \\
& \text { while CCM }>\varepsilon \text { do } \\
& \quad S_{1_{i, j}}=(1-\lambda) S_{1_{i, j}}+\frac{\lambda}{2}\left(b_{i, j+1}-b_{i, j}\right), j<L ; \\
& \quad S_{2_{i, j}}=(1-\lambda) S_{2_{i, j}}+\frac{\lambda}{2}\left(b_{i, M-L+j}-b_{i, M-L+j-1}\right), j<L ; \\
& B_{1}(x, y)=\left[S_{1, M \times L}(x, y), b(x, y), S_{2, M \times L}(x, y)\right] ; \\
& \hat{g}(x, y)=F^{-1}\left[B_{1}(x, y) / H(x, y)\right] ; \mathbf{R L} \text { algorithm } \\
& \min \mathrm{CCM}=\sum_{i=0}^{M-2}[\operatorname{ccm}(i, i+1)]^{2} ;
\end{aligned}
$$

end while

Output: deblurred image $\hat{g}(x, y)$.

\section{Experiment results}

The blur length is first obtained by the FFT method, then the deconvolution model is optimized by the $\alpha$-confidence method. In the experiment, this two-step image deblurring approach is verified with synthetic and standard images. It is also tested with the blurred images acquired from our rail-defect-detection system. It is demonstrated that this two -step deblurring approach can restore the image even with the serious motion blur.

\subsection{Synthetic and standard images deblurring}

The original image of Lena with the resolution of $1024 \times 1024$ pixels is shown in Fig. 4a. This image was degraded with serious blur by synthetic convolution in Fig. 4 b. We have compared many deblurring methods codes by the authors and their best results. Figure 4 lists several deblurring results with the relatively good visual quality. Parameters of our $\alpha$-confidence method are set with a unilateral confidence mean of $\alpha=0.01$ and an error permissible value $k=0.04$. Our two-step deblurring approach 

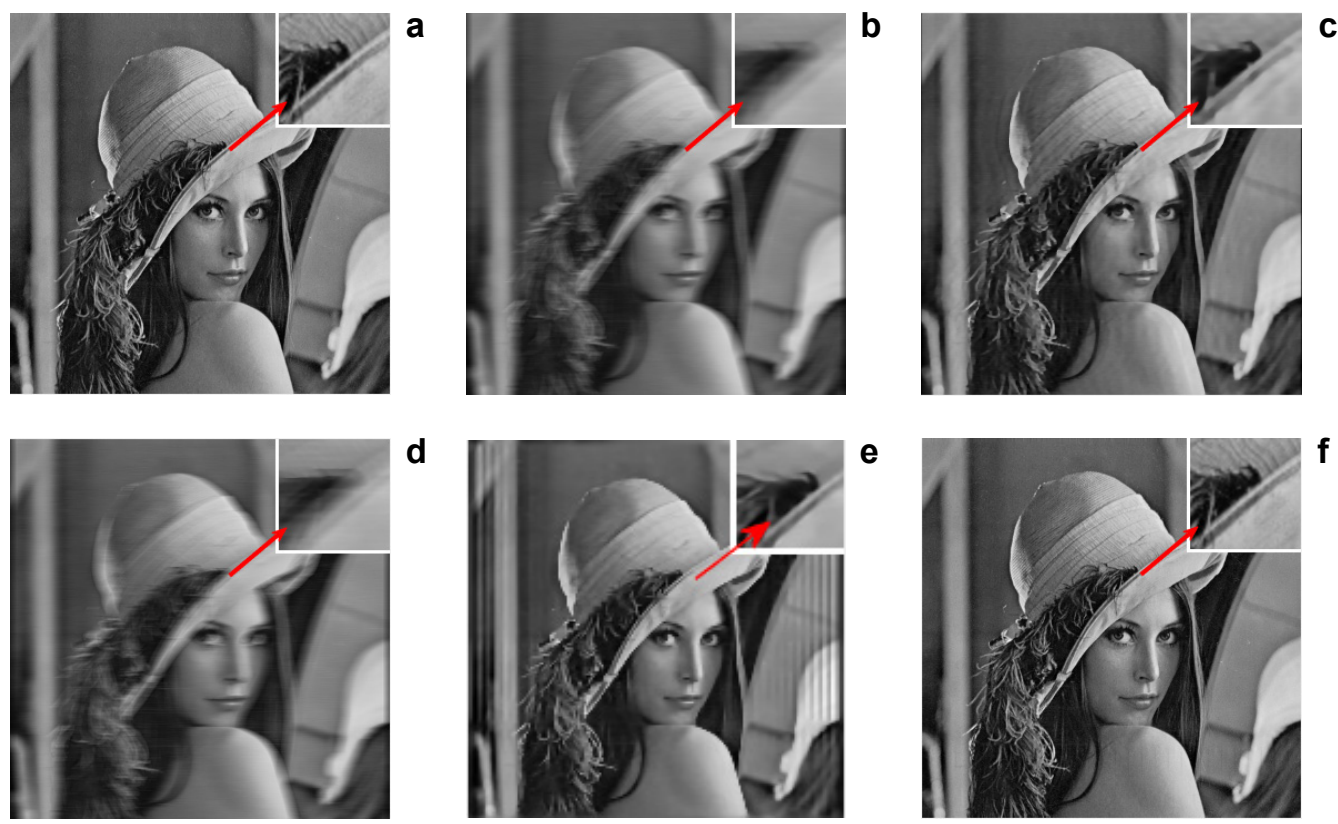

Fig. 4. The comparison of different deblurring methods. Original (a), blurred (b), AlmEIDA and Figueiredo [1] ] (c), Xu et al. [1] (d), ZHANG et al. []] (e), and ours (f).

acquires the sharp image details with fewer artifacts than the alternatives, as shown in Fig. 4.

Moreover, the robustness of our $\alpha$-confidence method under different blur parameters is compared in the Table, and the blurred images with $2 \%$ noise added. The image restoration quality is demonstrated by comparing the quality assessment indexes of the restored images and the blurred images. The quality assessment indexes such as the root mean squared (RMS) error, the signal to noise ratio (SNR) and the peak signal to noise ratio (PSNR) are employed to assess the restored image quality. Under normal circumstances, the higher values of the SNR and the PSNR indicate a better image quality, and the RMS value is the opposite. From these indexes, our $\alpha$-confidence method can restore images with better results even with large blur parameters. It is shown in

$\mathrm{T}$ a b 1 e. Blurred and restoration image quality with different blur parameters.

\begin{tabular}{|c|c|c|c|c|c|c|c|}
\hline \multirow{2}{*}{$\theta$} & \multirow{2}{*}{$L$ [pixels] } & \multicolumn{3}{|c|}{ Blurred images } & \multicolumn{3}{|c|}{ Restoration images } \\
\hline & & RMS & SNR & PSNR & RMS & SNR & PSNR \\
\hline $0^{\circ}$ & 5 & 5.565 & 1.881 & 33.222 & 1.112 & 9.423 & 47.212 \\
\hline $3^{\circ}$ & 25 & 7.601 & 1.370 & 30.513 & 2.085 & 5.024 & 41.750 \\
\hline $10^{\circ}$ & 50 & 7.826 & 1.323 & 30.260 & 2.532 & 4.137 & 40.061 \\
\hline $15^{\circ}$ & 75 & 7.830 & 1.316 & 30.167 & 2.664 & 3.932 & 39.621 \\
\hline $30^{\circ}$ & 100 & 7.861 & 1.308 & 30.033 & 2.745 & 3.816 & 39.359 \\
\hline
\end{tabular}




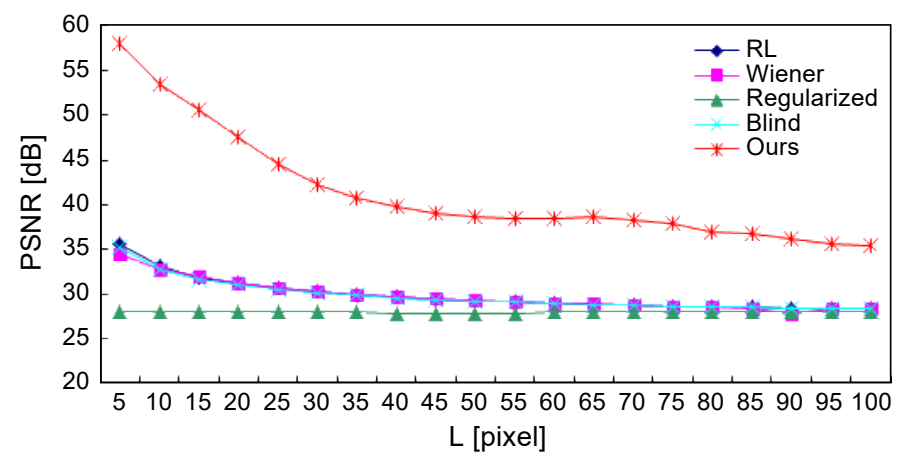

Fig. 5. Comparison of restoration quality with varying blur lengths and different methods.

the Table that the quality of the restored images is obviously better than the blurred images.

In Fig. 5, our $\alpha$-confidence restoration method is compared with other standard methods using the Matlab image restoration toolbox. The PSNR is used as the assessment of the image restoration quality, and the blur lengths set between 0 and 100 pixels. Experiment results obtained with different methods reveal that the PSNR values of the Wiener filtering method are better than that of the RL method at the medium blur lengths. Restoration results obtained by the Wiener filtering method, however, are not consistent across all tested lengths. The blind-image deconvolution method produces worse results than the Wiener filtering method and the RL method. The PSNR values of the regularized method remain unchanged with different blur lengths, thus, its sensitivity is the lowest of these methods. Therefore, the restoration quality with our $\alpha$-confidence deblurring method is significantly higher than the other methods from the PSNR values in Fig. 5.

\subsection{Application in fast motion deblurring}

The proposed two-step deblurring approach is then tested on real images of resolution of $2048 \times \times 1024$ pixels from our rail-defect-detection system [ $\underline{5}, \underline{6}]$. The camera system is attached to the inspection car with stabilization system and adequate light source. However, some of the acquired images show blurring due to the fast forward motion of the camera system, and the blurred ones are shown in Fig. 6.

The image in Fig. 6a is a blurred one from the inspection system with challenged blurs. Compared to other methods, our two-step deblurring approach obtains the sharp image as in Fig. 6f. Details from the enlarged image (see from the upper right corner in Fig. 6) show that our two-step approach can restore sharp boundary details better than other methods.

For the further verification, million blurred images were collected from our inspection system, and these images are deblurred by the proposed approach. It shows that if images are with small blurring, there is no significant difference of the deblurred 

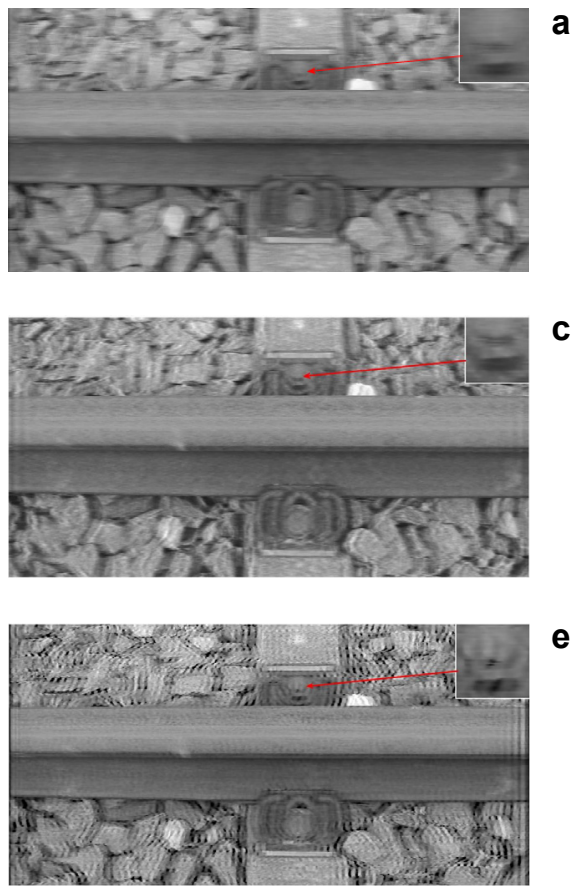

Fig. 6. Rail image deblurring. Blurred (a), RL [1ㅜ] (b), KRISHNAn et al. [16] (c), Xu et al. [18] (d), Sun et al. [19] (e), and ours (f).

image quality between our proposed approach and other methods. But for the serious blurring images, our proposed approach is better than others.

\section{Conclusion}

A two-step deblurring approach was proposed to restore the motion blur images, especially the serious blur ones, which has been applied to deblur the fast motion degraded images. A routine with two times of FFT was presented. The theoretical derivation and the experiment results show that the method can acquire an accurate estimation of blur lengths even in large blurs. The boundary prediction and the $\alpha$-confidence optimized methods were proposed for removing artifacts in the restored image, since the directly deconvolution of the real-image yields artifacts. Subjective visual perception and objective evaluation indexes of the image quality all indicate that our two-step deblurring approach can effectively improve the quality of motion blur images. The proposed image deblurring approach has been applied to our rail-defect detection system, and it can also be applied to other similar forward motion image deblurring.

Acknowledgments - This work was supported by the National Natural Science Foundation of China (Grant Nos. 51065021 and 51705229), and the Youth Scientific Foundation of Jiangxi Province (Grant No. 
20161BAB216128). We also highly appreciate Professor Jianhua $\mathrm{Wu}$ and anonymous reviewers for insightful and constructive comments.

\section{References}

[1] Cheong J.Y., Simon C., Kim C.-S., Park I.K., Reflection removal under fast forward camera motion, IEEE Transactions on Image Processing 26(12), 2017, pp. 6061-6073, DOI: 10.1109/TIP.2017.2748389.

[2] Ruiz P., Zhou X., Mateos J., Molina R., Katsaggelos A.K., Variational Bayesian blind image deconvolution: a review, Digital Signal Processing 47, 2015, pp. 116-127, DOI: 10.1016/j.dsp.2015. 04.012.

[3] Gong D., Tan M., Shi Q., van den Hengel A., Zhang Y., MPTV: matching pursuit-based total variation minimization for image deconvolution, IEEE Transactions on Image Processing 28(4), 2019, pp. 1851-1865, DOI: 10.1109/TIP.2018.2875352.

[4] Lu S., Liu Z., SHEN Y., Automatic fault detection of multiple targets in railway maintenance based on time-scale normalization, IEEE Transactions on Instrumentation and Measurement 67(4), 2018, pp. 849-865, DOI: 10.1109/TIM.2018.2790498.

[5] Yuan X., Wu L., Peng Q., An improved Otsu method using the weighted object variance for defect detection, Applied Surface Science 349, 2015, pp. 472-484, DOI: 10.1016/j.apsusc.2015.05.033.

[6] Huang L.E., Wu L.S., Cheng H.W., Image blur types and parameters estimation using DCNN fusion with the LSTM, Journal of Basic Science and Engineering 26(5), 2018, pp. 1092-1100.

[7] Zhang J., PAN J., LAI W.-S., LAU R.W.M., YANG M.-H., Learning fully convolutional networks for iterative non-blind deconvolution, IEEE Conference on Computer Vision and Pattern Recognition, July 2017, Hawaii, USA, pp. 3817-3825, DOI: 10.1109/CVPR.2017.737.

[8] Deshrande A.M., Patnaik S., A novel modified cepstral based technique for blind estimation of motion blur, Optik 125(2), 2014, pp. 606-615, DOI: 10.1016/j.ijleo.2013.05.189.

[9] Chang M.M., Tekalp A.M., ERdem A.T., Blur identification using the bispectrum, IEEE Transactions on Signal Processing 39(10), 1991, pp. 2323-2325, DOI: 10.1109/78.91207.

[10] LuCy L.B., An iterative technique for the rectification of observed distributions, The Astronomical Journal 79(6), 1974, pp. 745-754.

[11] Baselice F., Ferraioli G., Ambrosanio M., Pascazio V., Schirinzi G., Enhanced Wiener filter for ultrasound image restoration, Computer Methods and Programs in Biomedicine 153, 2018, pp. 71-81, DOI: $10.1016 / \mathrm{j} . \mathrm{cmpb} .2017 .10 .006$.

[12] Bentahar Y., Afifi M., Dalimi H., Amar S., Restoration image degraded by a blurred variable in the field, Optica Applicata 48(1), 2018, pp. 5-14, DOI: 10.5277/oa180101.

[13] Demircan-Tureyen E., Kamasak M.E., Directional total variation based image deconvolution with unknown boundaries, [In] Computer Analysis of Images and Patterns. CAIP 2017, [Eds.] Felsberg M., Heyden A., Krüger N., Lecture Notes in Computer Science, Vol. 10425, Springer, Cham, 2017, pp. 473-484, DOI: 10.1007/978-3-319-64698-5_40.

[14] Almeida M.S.C., Figueiredo M., Deconvolving images with unknown boundaries using the alternating direction method of multipliers, IEEE Transactions on Image Processing 22(8), 2013, pp. 3074-3086, DOI: $10.1109 /$ TIP.2013.2258354.

[15] BAi Y., Cheung G., Liu X., GaO W., Graph-based blind image deblurring from a single photograph, IEEE Transactions on Image Processing 28(3), 2019, pp. 1404-1418, DOI: 10.1109/TIP.2018.2874290.

[16] Krishnan D., TAY T., Fergus R., Blind deconvolution using a normalized sparsity measure, IEEE Conference on Computer Vision and Pattern Recognition, June 2011, Colorado, USA, pp. 233-240, DOI: 10.1109/CVPR.2011.5995521.

[17] Levin A., Weiss Y., Durand F., Freeman W.T., Efficient marginal likelihood optimization in blind deconvolution, IEEE Conference on Computer Vision and Pattern Recognition, June 2011, Colorado, USA, pp. 2657-2664, DOI: 10.1109/CVPR.2011.5995308. 
[18] Xu L., ZHeNG S., JiA J., Unnatural $L_{0}$ sparse representation for natural image deblurring, IEEE Conference on Computer Vision and Pattern Recognition, June 2013, Oregon, USA, pp. 1107-1114, DOI: 10.1109/CVPR.2013.147.

[19] SUN J., CAO W., XU Z., PONCE J., Learning a convolutional neural network for non-uniform motion blur removal, IEEE Conference on Computer Vision and Pattern Recognition, June 2015, Boston, Massachusetts, pp. 769-777, DOI: 10.1109/CVPR.2015.7298677.

[20] HAN Y., KAN J., Blind color-image deblurring based on color image gradients, Signal Processing 155, 2019, pp. 14-24, DOI: 10.1016/j.sigpro.2018.09.032.

[21] Shamik Tiwari, Shukla V.P., Singh A.K., Biradar S.R., Review of motion blur estimation techniques, Journal of Image and Graphics 1(4), 2013, pp. 176-184, DOI: 10.12720/joig.1.4.176-184.

[22] WAng Z., Yao Z., Wang Q., Improved scheme of estimating motion blur parameters for image restoration, Digital Signal Processing 65, 2017, pp. 11-18, DOI: 10.1016/j.dsp.2017.02.010.

[23] Yuan L., Sun J., QuAn L., Shum H.-Y., Image deblurring with blurred/noisy image pairs, ACM Transactions on Graphics 26(3), 2007, article 1, DOI: 10.1145/1276377.1276379.

[24] Shan Q., Jia J., Agarwala A., High-quality motion deblurring from a single image, ACM Transactions on Graphics 27(3), 2008, article 73, DOI: 10.1145/1360612.1360672.

[25] Chan S.H., Wang X., Elgendy O.A., Plug-and-play ADMM for image restoration: fixed-point convergence and applications, IEEE Transactions on Computational Imaging 3(1), 2017, pp. 84-98, DOI: $10.1109 /$ TCI.2016.2629286.

[26] Mosleh A., Sola Y.E., Zargari F., Onzon E., Langlois J.M.P., Explicit ringing removal in image deblurring, IEEE Transactions on Image Processing 27(2), 2018, pp. 580-593, DOI: 10.1109/TIP. 2017.2764625.

[27] Cao S., He N., Zhao S., Lu K., Zhou X., Single image motion deblurring with reduced ringing effects using variational Bayesian estimation, Signal Processing 148, 2018, pp. 260-271, DOI: $10.1016 /$ j.sigpro.2018.02.015.

[28] Shao W.-Z., Deng H.-S., Ge Q., Li H.-B., Wei Z.-H., Regularized motion blur-kernel estimation with adaptive sparse image prior learning, Pattern Recognition 51, 2016, pp. 402-424, DOI: 10.1016/ i.patcog.2015.09.034.

[29] Zноu N.R., Luo A.W., Zou W.P., Secure and robust watermark scheme based on multiple transforms and particle swarm optimization algorithm, Multimedia Tools and Applications 78(2), 2019, pp. 2507 -2523, DOI: 10.1007/s11042-018-6322-9.

[30] Abramovich F., Ritov Y., Statistical Theory: A Concise Introduction, CRC Press, Florida, USA 2013, pp. 121-129.

Received March 21, 2019

in revised form May 20, 2019 